\title{
Genome-wide identification and characterization of the superoxide dismutase gene family in Musa acuminata cv. Tianbaojiao (AAA group)
}

Xin Feng, Zhongxiong Lai, Yuling Lin, Gongti Lai and Conglong Lian

\begin{abstract}
Background: Superoxide dismutase (SOD) is an essential enzyme of the plant antioxidant system that responds to oxidative stresses caused by adverse conditions. Banana is an important staple and economic crop in tropical and subtropical regions. However, its growth and yield are constantly affected by various abiotic stresses. To analyze the roles of distinct SOD genes under various stresses, a detailed characterization and analysis of the SOD gene family in Cavendish banana is indispensable.
\end{abstract}

Methods: The presence and structure of the SOD family genes were experimentally verified using 5'/3' RACE-PCR, reverse transcription PCR and PCR. Then, their syntenic relationships, conserved motifs and phylogenetic relationships were analyzed using software. Cis-elements present in the promoters were predicted via PlantCARE. And the expression levels under abiotic and hormonal stresses were determined using real-time quantitative polymerase chain reaction.

Results: In total, 25 Tianbaojiao' SOD cDNAs (MaSODs), which encoded six Cu/ZnSODs, four MnSODs and two FeSODs, were cloned. The 12 MaSOD genes were divided into four groups based on their conserved motifs, which corroborated their classifications based on gene-structure patterns and subcellular localizations. Eleven MaSOD promoters were isolated and found to contain many cis-acting elements involved in stress responses. Gene expression analysis showed that 11 out of the 12 MaSODs were expressed in all tested tissues (leaf, pseudostem and root), whereas MaCSD2B was expressed only in leaves and roots. Specific MaSOD members exhibited different expression patterns under abiotic and hormonal treatments. Among the 12 MaSOD genes, MaCSD1D was the only one that responded to all eight treatments, suggesting that this gene plays a predominant role in reactive oxygen species scavenging caused by various stresses in banana.

Conclusions: A genome-wide analysis showed that the 'Tianbaojiao' banana harbored an expanded SOD gene family. Whole genome duplication, segmental duplication and complex transcriptional regulation contributed to the gene expansion and mRNA diversity of the MaSODs. The expression patterns of distinct MaSOD genes showed that they are important responses to different abiotic and hormonal stresses in banana.

Keywords: Musa acuminata cv. Tianbaojiao (AAA group), SOD gene family, Promoter, Abiotic stress, Hormonal stress, Expression analysis

\footnotetext{
*Correspondence: laizx01@163.com

Institute of Horticultural Biotechnology, Fujian Agriculture and Forestry

University, Fuzhou, Fujian 350002, China
}

(c) 2015 Feng et al. Open Access This article is distributed under the terms of the Creative Commons Attribution 4.0 International License (http://creativecommons.org/licenses/by/4.0/), which permits unrestricted use, distribution, and reproduction in any medium, provided you give appropriate credit to the original author(s) and the source, provide a link to the Creative Commons license, and indicate if changes were made. The Creative Commons Public Domain Dedication waiver (http://creativecommons.org/publicdomain/zero/1.0/) applies to the data made available in this article, unless otherwise stated. 


\section{Background}

Banana is an important staple and economic crop in tropical and subtropical regions. However, its growth and yield are constantly affected by severe abiotic and biotic stresses, such as cold in winter, drought and water-logging, as well as various diseases and pests [1]. These environmental perturbations often lead to the increased generation of reactive oxygen species (ROS) in plant cells [2]. Excess ROS can attack virtually all cellular macromolecules. This usually results in membrane damage, protein oxidation and DNA lesions, and can even lead to irreparable metabolic dysfunction and cell death $[3,4]$. Thus, to cope with ROS toxicity, plants have developed efficient and complex antioxidative response systems, including many non-enzymatic and enzymatic components. Among these enzymatic components, superoxide dismutases (SODs), acting as the first line of antioxidant systems in plant, play important roles in catalyzing the dismutation of superoxide radicals to protect cells from oxidative damage [5].

In plants, there exist multiple SOD isozymes, which are classified into three types based on their metal cofactors: $\mathrm{Cu} / \mathrm{ZnSOD}$, FeSOD and MnSOD [6]. Although these SOD proteins are encoded by nuclear genes, they are distributed to different cellular compartments. $\mathrm{Cu}$ / ZnSODs are mainly located in the cytosol, chloroplasts, peroxisomes and/or the extracellular space, while FeSODs are mainly in chloroplasts and possibly the cytosol, and MnSODs are in the mitochondria [7]. Owing to their important roles in the antioxidant system, a considerable number of $S O D$ genes are cloned from various monocot and dicot plants [8-12]. However, Arabidopsis thaliana and Populus trichocarpa are the only two plants whose $S O D$ gene families have been characterized in the genome-wide level at present, and the numbers of the three $S O D$-type genes varies among them $[8,9]$.

$S O D s$ contribute to various environmental stimuli responses in plants, such as cold, drought, salinity, auxin and ethylene [7, 13, 14]. Different SOD genes exhibited different expression patterns. The responses of SODs to environmental changes or stresses were dramatically different, depending on the different SOD members present, the stress and the species. For instance, under ozone fumigation, the transcriptional levels of chloroplastic CSD2 and FSD1 were transiently decreased, while chloroplastic FSD2 mRNAs remained somewhat constant in A. thaliana [8]. In contrast, FSD2 mRNAs dramatically increased in response to UV-B, while CSD2 or FSD1 mRNAs remained constant. In addition, cytosolic CSD1 could be involved in responses to both ozone fumigation and UV-B illumination. Even so, the $S O D$ genes of the same $S O D$ type did not always exhibit uniform functions in different species. MnSOD showed no altered expression when subjected to a series of oxidative stress treatments in Arabidopsis, but it responded positively to salt stress in pea [15], and cold and drought stress in wheat $[16,17]$. This indicated that the regulation of $S O D$ genes is complicated in response to oxidative stress. Cis-element and transcription factor analyses of SOD promoters from Arabidopsis [6], wheat [12] and longan [10] provided some clues on how the SOD genes are modulated. Additionally, alternative splicing (AS) and miRNAs have also found to be involved in the regulation of $S O D$ expression [18, 19]. Studies using over-expressing or knocked-out plant $S O D$ genes have confirmed their functions in improving stress tolerance [20-22].

In previous reports, Zhou et al. studied the SOD isoenzymes in banana using biochemical methods and revealed that cold stress led to the accumulation of different SOD isoenzymes [23]. A quantitative proteomic analysis confirmed the existence of $\mathrm{Cu} / \mathrm{ZnSOD}, \mathrm{MnSOD}$ and FeSOD in banana [24]. However, these studies focused only on the proteins and changes in activity, which were unable to effectively elucidate the exact roles of banana SODs under adverse conditions. Recently, the whole-genome sequences of Musa acuminata var. DHPahang (wild banana, AA group) and Musa balbisiana var. Pisang Klutuk Wulang (PKW; wild banana, BB group) were made available to the public [25, 26], facilitating molecular studies on the expression and regulatory mechanisms of banana SODs in response to oxidative stress. Using these genomes, we performed a genome-wide identification of the $S O D$ gene family in M. acuminata cv. Tianbaojiao (Cavendish banana, AAA group) to analyze the transcript types, protein motifs, exon-intron organizations, chromosomal locations and phylogenetic relationships. Then, the putative promoters of banana SODs were also isolated and cis-elements involved in stress responses were analyzed to further illuminate their transcriptional regulatory mechanisms. Finally, we studied the expression profiles of MaSOD family genes under abiotic (cold, heat, drought and salt) and hormonal [abscisic acid (ABA), gibberellin $\mathrm{A}_{3}$ $\left(\mathrm{GA}_{3}\right)$, indole-3-acetic acid (IAA) and salicylic acid (SA)] stresses using real-time quantitative polymerase chain reaction ( $\mathrm{qPCR}$ ), which should help determine the functions of each MaSOD gene under adverse conditions.

\section{Methods}

Plant materials and stress treatments

Sterile plantlets of $M$. acuminata cv. Tianbaojiao (Cavendish banana, AAA group) were generated by inducing suckers as Zhang et al. described [27]. Twentyfive-day-old sterile plantlets were used in abiotic stress treatments, except the drought treatment. The plantlets were cultivated in Murashige and Skoog liquid solution with $200 \mathrm{mM} \mathrm{NaCl}$ for salt treatment, $100 \mu \mathrm{M}$ IAA, $100 \mu \mathrm{M} \mathrm{GA}{ }_{3}$ and $100 \mu \mathrm{M}$ SA for hormonal treatments, 
and sprayed with $100 \mu \mathrm{M}$ ABA in $0.02 \%(\mathrm{v} / \mathrm{v})$ Tween 20 for the ABA treatment. These treated plantlets were sampled at 4, 8, 12, 24 and $48 \mathrm{~h}$, except that salt treatment, which was sampled at 4, 12, 24, 48 and 72 h. For the drought treatment, 2-month-old plants grown in soil were cultivated without watering and sampled at $1 \mathrm{~d}, 2 \mathrm{~d}, 3 \mathrm{~d}, 4$ $\mathrm{d}$ and $5 \mathrm{~d}$. All the treatment conditions were at $28{ }^{\circ} \mathrm{C}$ with 3300 lux continuous light, except the cold stress, which was conducted in $4{ }^{\circ} \mathrm{C}$ growth chambers with 400 lux continuous light, and the heat stress, which was conducted in $40{ }^{\circ} \mathrm{C}$ growth chambers with 3300 lux continuous light. Control and treated plantlets were immediately frozen in liquid nitrogen and stored at $-80{ }^{\circ} \mathrm{C}$ until nucleic acid extraction.

\section{Nucleic acid extraction and CDNA synthesis}

Genomic DNAs were extracted from 'Tianbaojiao' banana using the modified CTAB method [28]. Total RNAs were

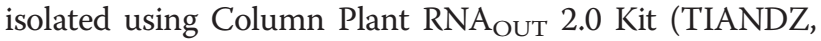
China). Then, the quality was checked using $1.0 \%$ agarose gel electrophoresis and quantified using spectrophotometry. Total RNAs were reverse transcribed using a Thermo Scientific RevertAid First Strand cDNA Synthesis Kit (Fermentas, EU) for 3' UTR (untranslated region) and open reading frame (ORF) cloning, and a SMART ${ }^{\text {Tm }}$ RACE cDNA Amplification kit (Takara, Japan) for 5' UTR cloning. A GeneRacer ${ }^{\text {Tw }}$ kit (Invitrogen, USA) was used for the alternative transcriptional start site analysis.

\section{Isolation of the SOD genes from M. acuminata cv. Tianbaojiao}

"Superoxide dismutase" and published SOD sequences in NCBI were used as the index words and probes, respectively, to search for $S O D$ genes in the wild banana genome databases (http://banana-genome.cirad.fr/) [29]. Full-length cDNAs of 'Tianbaojiao' SOD genes were cloned using 5'/3' RACE-PCR and reverse transcription PCR (RT-PCR), with primers designed from known $S O D$ sequences in the NCBI and the banana genome databases. Genomic sequences of MaSODs were obtained from DNA templates using PCR. 5' - flanking regions of MaSOD genes, $1.0-2.0 \mathrm{~kb}$ in length, were generated using PCR with forward primers (designed to regions $2.0 \mathrm{~kb}$ upstream of the start codon of 'DH-Pahang' SOD genes) and reverse primers (designed to regions downstream of the start codon of each MaSOD). All of the primer information is listed in Additional files 1 and 2 .

PCR reactions were performed according to the instructions of ThermoScientific DreamTaq Green PCR Master Mix $(2 \times)$ (USA) and Takara LA Taq (Japan). Then, PCR products with acceptable sizes were purified, subcloned into the vector pMD18-T (Takara, Japan), and confirmed by DNA sequencing. The correct sequences of $S O D$ genes were abbreviated as MaCSD, MaMSD and
MaFSD for $\mathrm{Cu} / \mathrm{ZnSOD}, \mathrm{MnSOD}$ and FeSOD, respectively, with a prefix representing the genus and species.

\section{Sequence analysis and subcellular localization}

Multiple sequence alignments were performed using ClustalX (version 1.83). The molecular weights and isoelectric points of the MaSODs were calculated using the ExPASy ProtParam tool [30]. Syntenic relationships of SOD genes were searched in the Plant Genome Duplication Database (PGDD, http://chibba.agtec.uga.edu/duplication/) [31]. Conserved motifs were detected using MEME with the default settings, except that the maximum number of motifs was defined as nine, and the minimum and maximum motif width was set to 20 and 150, respectively. Exon-intron organizational analyses were carried out using the Gene Structure Display Server (http://gsds2.cbi.pku. edu.cn/). Transcriptional response elements of the promoters were predicted using the PlantCARE tool [32].

Subcellular localizations and putative transit peptides were predicted by SoftBerry (http://linux1.softberry.com/), ChloroP1.1 (http://www.cbs.dtu.dk/services/ChloroP/) [33] and MITOPROT (http://ihg.gsf.de/ihg/mitoprot.html) [34]. The coding regions of $M a C S D 1 D$ were amplified using specific primers. The forward primer was 5'CATGCCATG GATGGTTAAGGCTGTAGCTGTG-3' and the reverse primer was 5'-GGACTAGTCTCCTGAAGCCCAATGAC AC-3' (restriction enzyme sites are in bold). The amplicons were then ligated into the $\mathrm{N}$-terminus of the green fluorescent protein (GFP) sequence of the pCAMBIA1302 vector to generate the pCAMBIA1302-35S::MaCSD1D-GFP::NOS construct. The recombinant plasmid was introduced into onion epidermal cells by Agrobacterium-mediated transformation according to a reported method [35]. Two days later, the subcellular localization of the MaCSD1D protein was detected using an A1R/A1 laser confocal scanning microscope (Nikon, Japan).

\section{Chromosomal locations and phylogenetic analysis}

MaSOD genes were mapped to the chromosomes by performing a BLASTn search against the banana genome databases. Unrooted phylogenetic trees based on $S O D$ protein sequences were constructed using the maximum likelihood method of the MEGA 5.02 software with the Poisson model and a bootstrap of 1000 replicates [36].

\section{Real-time quantitative PCR and data analysis}

Total RNAs from different tissues (leaf, pseudostem and root) and stress-treated leaves were reverse transcribed with PrimeScript $^{\text {ti }}$ RT Master Mix (Perfect Real Time) kit (Takara, Japan) into cDNAs for qPCR analysis. Primers, specific to every MaSOD gene, were designed based on the specific 3' UTR sequences, except that of MaFSD1B, which were designed on the $5^{\prime}$ end sequences. A clathrin 
adaptor complexes medium gene $(\mathrm{MaCAC})$ was used as an internal control because of its relatively stable expression level [37]. The specificity of primers was tested and verified by analyzing the melting curve. A gene expression analysis was performed using a LightCycler480 Real-time PCR detection instrument (Roche, Switzerland) and SYBR $^{\circ}$ Premix Ex Taq ${ }^{\text {Tix }}$ II (Tli RNaseH Plus; Takara, Japan) with three biological replicates and technical replicates. PCR reactions included an initial denaturation at $95^{\circ} \mathrm{C}$ for $3 \mathrm{~min}$, followed by 40 cycles at $95^{\circ} \mathrm{C}$ for $10 \mathrm{~s}, 57^{\circ} \mathrm{C}$ for $20 \mathrm{~s}$, and $72{ }^{\circ} \mathrm{C}$ for $30 \mathrm{~s}$. Relative expression levels were determined using the $2^{-\Delta \Delta \mathrm{Ct}}$ method. Then, the expression patterns of the $12 \mathrm{MaSOD}$ genes were clustered by $\mathrm{MeV}$ (version 4.8) using the average linkage hierarchical clustering method. The details of the specific primers for qPCR are listed in Additional file 3.

\section{Results}

Identification of the SOD gene family in M. acuminata cv. Tianbaojiao

In the wild banana genome databases, 15 sequences in 'DH-Pahang' (AA group) and 14 sequences in 'PKW' (BB group) were identified in silico as $S O D$ genes based on annotations and a BLASTn search using known $S O D$ genes from NCBI. Excluding the remnant sequences and chimeric genes, there were 13 potentially functional $S O D$ genes in 'DH-Pahang' and 11 genes in 'PKW' (Additional file 4). Two of these genes (GSMU_Achr10T27190_001 and GSMU_Achr10T27220_001 of 'DH-Pahang', and ITC1587_Bchr10_T31275 and ITC1587_Bchr10_T31280 of ' $\mathrm{PKW}$ ') were tandemly located on chromosome 10 and shared similar ORF and gDNA sequences, which indicates that they were recently tandemly duplicated. Sequence analysis revealed that the $S O D$ ORF lengths and sequence identities of the two wild bananas were somewhat different (Additional file 4). In this study, the presence and the structure of the $S O D$ family genes were experimentally verified in the triploid cultivated banana 'Tianbaojiao'.

A total of 25 different $S O D$ cDNAs with intact ORFs were obtained from banana 'Tianbaojiao' using 5' and 3' RACE-PCR and confirmed by RT-PCR. Two of the cDNAs were described previously [38] and renamed here as MaCSD1A-1 (GenBank: JQ411718) and MaCSD1A-2 (GenBank: JQ411719) for the sake of consistency. A sequence alignment and BLAST results using known SODs/ SODs in the NCBI database as queries revealed that these 25 transcripts were transcribed from 12 different MaSOD genes as a result of alternative transcription start sites (ATSSs), AS and alternative polyadenylation (APA). These transcripts, which encoded the same SOD protein, shared an identical ORF but differed in their UTRs. The major MaSOD features are summarized in Table 1.

The ORF of MaCSD1A-1 was $98.14 \%$ identical to that of $M a C S D 1 A-2$. A further analysis of their corresponding
gDNAs (GenBank: KM017525 and KM017524) revealed they were alleles. Three independent transcripts encoding MaCSD1B, named MaCSD1B-1,-2 and -3, shared the same 459 bp ORF but varied in the 5' UTR length or nucleotide composition owing to AS and ATSSs (Fig. 1a-1). Two other transcripts contained a 459-bp ORF but encoded two different amino acid polypeptides, which were named as $M a C S D 1 C$ and $M a C S D 1 D$. The two transcripts, designated as MaCSD2A-1 and MaCSD2A-2, contained the same ORF of 684 bp but with different $3^{\prime}$ and 5' UTRs (Fig. 1a-2 and b-1). The 996-bp long cDNA $M a C S D 2 B$ had a 675-bp ORF with a 45-bp 5' UTR and a 276-bp 3' UTR.

Ten independent transcripts of $M n S O D$ were classified into four MaMSD genes (named MaMSD1A, 1B, 1C and 1D) based on the sequence differences of their ORFs and deduced amino acids. Each MaMSD gene contained two to four types of transcripts with different 3' UTR lengths (Fig. 1b-2, b-3 and b-4), resulting from APA, except MaMSD1D, which had a unique 3' UTR (Table 1). APA sites were also found in the MnSOD genes of Larix gmelinii [39].

Multiple sequence alignments of the five MaFSD transcripts showed that they encoded two FeSOD genes with distinct ORFs. MaFSD1A-1,-2 and -3 , co-encoded a 301-amino acid polypeptide, contained the same $906 \mathrm{bp}$ ORF but had different 3' UTR lengths (Fig. 1b-5). The other two cDNAs shared $100 \%$ identity, except that one of them contained two extra sequences of $87 \mathrm{bp}$ and $172 \mathrm{bp}$ in the ORF region (Additional file 5). Aligning with gDNA sequences showed that these two extra sequences were introns retained in the mRNA during splicing, forming an alternative splicing transcript named MaFSD1B-variant1. Comparing MaFSD1B-variant1 with the normal transcript MaFSD1B revealed that its ORF was shorter with a premature stop codon.

In addition, the isoelectric points of the $12 \mathrm{MaSOD}$ proteins ranged from 4.87 to 7.90 , and their molecular weights varied from 15.0 to $34.2 \mathrm{kDa}$ (Table 1). Pairwise similarities among the $12 \mathrm{MaSOD}$ genes were performed and are listed in Additional file 6. The analysis revealed that the MaMSD genes shared $80.9-86.5 \%$ identity at both the nucleotide and deduced amino acid levels, followed by MaCSD genes at 39.7-87.5 \% and MaFSD genes at $33.3-42.5 \%$, whereas the similarity between genes from different MaSOD types, such as MaCSD1A and MaMSD1A, was below $35.6 \%$. A BLASTp search of the NCBI database revealed that the putative polypeptides of the MaSOD genes shared about $62.0-89.0 \%$ sequence identity with orthologous SOD proteins in other plants.

Intragenome syntenic relationship analysis indicated that $M a C S D 2 A$ and $2 B$, and MaMSD1A and $1 C$ or $1 D$ are derived from banana whole genome duplications 
Table 1 Characteristics of the SOD genes from M.acuminata CV. Tianbaojiao

\begin{tabular}{|c|c|c|c|c|c|c|c|c|c|}
\hline \multirow[t]{2}{*}{ Gene name } & \multicolumn{4}{|l|}{ CDNA } & \multicolumn{3}{|l|}{ Protein } & \multicolumn{2}{|l|}{ gDNA } \\
\hline & Accession NO. & $5^{\prime} \cup T R, b p$ & $3^{\prime} U T R^{a}, b p$ & ORF,bp & Length,aa & Molecular weight,kDa & $\mathrm{pl}$ & Length $^{\mathrm{b}}$,bp & Accession NO. \\
\hline MaCSDIA-1 & JQ411718 & 11 & 210 & 483 & 160 & 16.4 & 6.82 & 2891 & KM017525 \\
\hline MaCSDIA-2 & JQ411719 & 14 & 205 & 483 & 160 & 16.3 & 6.85 & 2863 & KM017524 \\
\hline MaCSD1B-1 & JX948788 & 134 & 208 & 459 & 152 & 15.2 & 5.47 & 2854 & KM017526 \\
\hline MaCSDIB-2 & KM017523 & 82 & - & 459 & 152 & 15.2 & 5.47 & 2854 & KM017526 \\
\hline MaCSD1B-3 & KM017516 & 79 & - & 459 & 152 & 15.2 & 5.47 & 2854 & KM017526 \\
\hline MaCSDIC & JX948789 & 66 & 184 & 459 & 152 & 15.0 & 5.82 & 2341 & KM017514 \\
\hline MaCSDID & KC007552 & 65 & 187 & 459 & 152 & 15.5 & 4.87 & 1807 & KM017512 \\
\hline MaCSD2A-1 & JX519461 & 82 & 227 & 684 & 227 & 23.1 & 6.11 & 4651 & KM017515 \\
\hline MaCSD2A-2 & KM017517 & 22 & 167 & 684 & 227 & 23.1 & 6.11 & 4651 & KM017515 \\
\hline MaCSD2B & KJ739805 & 45 & 276 & 675 & 224 & 22.7 & 7.16 & 3887 & KM017513 \\
\hline MaMSDIA-1 & JX519462 & 27 & 194 & 717 & 238 & 26.3 & 7.09 & 4447 & KM017530 \\
\hline MaMSDIA-2 & KM017528 & - & 156 & 717 & 238 & 26.3 & 7.09 & 4447 & KM017530 \\
\hline MaMSD1A-3 & KM017536 & - & 141 & 717 & 238 & 26.3 & 7.09 & 4447 & KM017530 \\
\hline MaMSD1A-4 & KM017529 & - & 116 & 717 & 238 & 26.3 & 7.09 & 4447 & KM017530 \\
\hline MaMSDIB-1 & JQ364939 & 65 & 177 & 732 & 243 & 26.5 & 6.75 & 3030 & KM017531 \\
\hline MaMSDIB-2 & KM017534 & - & 156 & 732 & 243 & 26.5 & 6.75 & 3030 & KM017531 \\
\hline MaMSD1B-3 & KM017535 & - & 112 & 732 & 243 & 26.5 & 6.75 & 3030 & KM017531 \\
\hline MaMSDIC-1 & JX844024 & 25 & 233 & 738 & 245 & 26.8 & 7.90 & 4720 & KM017532 \\
\hline MaMSD1C-2 & KM017527 & - & 213 & 738 & 245 & 26.8 & 7.90 & - & - \\
\hline MaMSD1D & KJ739806 & 44 & 223 & 732 & 243 & 26.4 & 6.76 & 3121 & KM017533 \\
\hline MaFSDIA-1 & JX535809 & 26 & 245 & 906 & 301 & 34.2 & 5.66 & 4080 & KM017518 \\
\hline MaFSD1A-2 & KM017520 & - & 223 & 906 & 301 & 34.2 & 5.66 & 4080 & KM017518 \\
\hline MaFSDIA-3 & KM017521 & - & 189 & 906 & 301 & 34.2 & 5.66 & 4080 & KM017518 \\
\hline MaFSDIB & KJ739807 & 70 & 453 & 783 & 260 & 29.9 & 6.75 & 4208 & KM017519 \\
\hline MaFSD1B-variant1 & KM017522 & - & - & 687 & 228 & 26.2 & 7.01 & 3853 & KM017519 \\
\hline
\end{tabular}

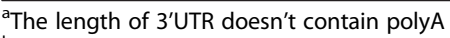

${ }^{\mathrm{b}}$ The gDNA is corresponding to the ORF of MaSODs

(WGDs). Cross-genome syntenic analysis revealed that another five MaSOD genes (MaCSD1A, MaCSD1D, MaMSD1B, MaFSD1A and MaFSD1B) are contained in blocks shared among different plant species, suggesting that the other duplicated copies of them in banana genome are lost after WGDs.

\section{Conserved motifs and clustering analysis of MaSODs}

To elucidate the domain features and phylogenetic relationship of the MaSODs, an unrooted phylogenetic tree and a linear distribution map of the conserved motifs in the deduced MaSODs were generated (Fig. 2). MaSODs were clustered into three major clades, which showed a good accordance with their metal cofactor types. The three clades were designated as groups I, II and III. Group I contained two subgroups (Ia and Ib) and harbored motif 1 and motif 5 , which contained $\mathrm{Cu} / \mathrm{ZnSOD}$ signatures and conserved $\mathrm{Cu}^{2+}$ and $\mathrm{Zn}^{2+}$ binding sites (Additional file 7). Compared with Group Ia, Group Ib included an additional motif 8, which contained chloroplast peptide signal sequences, suggesting MaCSD2A and 2B were located in chloroplasts, whereas MaCSD1A-1D were cytosolic $\mathrm{Cu} / \mathrm{ZnSODs}$ as predicted by SoftBerry. The MaCSD1D protein fused with GFP to co-express on onion epidermal cells, confirming the cytosolic localization (Fig. 3). Four MaMSDs formed group II, which contained the motifs of 2, 3 and 6. Motif 2 included the conserved metal-binding domain "DVWEHAYY" and five residues (Gly, Gly, Phe, Gln and Asp) [9] that were present in MnSOD but absent from FeSOD, while motif 6 included mitochondrial location signal sequences, suggesting they targeted to the mitochondria. Group III was made up of MaFSD1A and MaFSD1B, which shared motifs 3, 4 and 7. Residues (Ala, Gln, Trp, Phe and Ser) responsible for recognizing iron ion and active sites for FeSOD [40] were found in motif 4 and the conserved metal-binding domain "DVWEHAYY" was detected in motif 7. 


\section{A A-1 MaCSD1BgDNA MaCSD1B-1 MaCSD18-2 MaCSD18-3 MaCSD1B gDNA MaCSD18-1 MaCSD1B-2 MaCSD18-3 MaCSD1B gDNA MaCSD18-1 $\mathrm{MaCSD1B}-2$ MaCSD18 gDNA MaCSD18-1 MaCSD18-3 \\ A-2 MaCSD2A gDNA MaCSD2A-2 \\ A-3 MaCSD1C gDNA MaCSD1C-1 MaCSD1C gDNA MaCSD1C-1 \\ A-4 MaCSD1D gDNA MaCSD1D-1 MaCSD10 gDNA MaCSD1D-1 MaCSD1D gDNA MaCSD1D-1 MaCSD1D gDNA MaCSD1D-1}
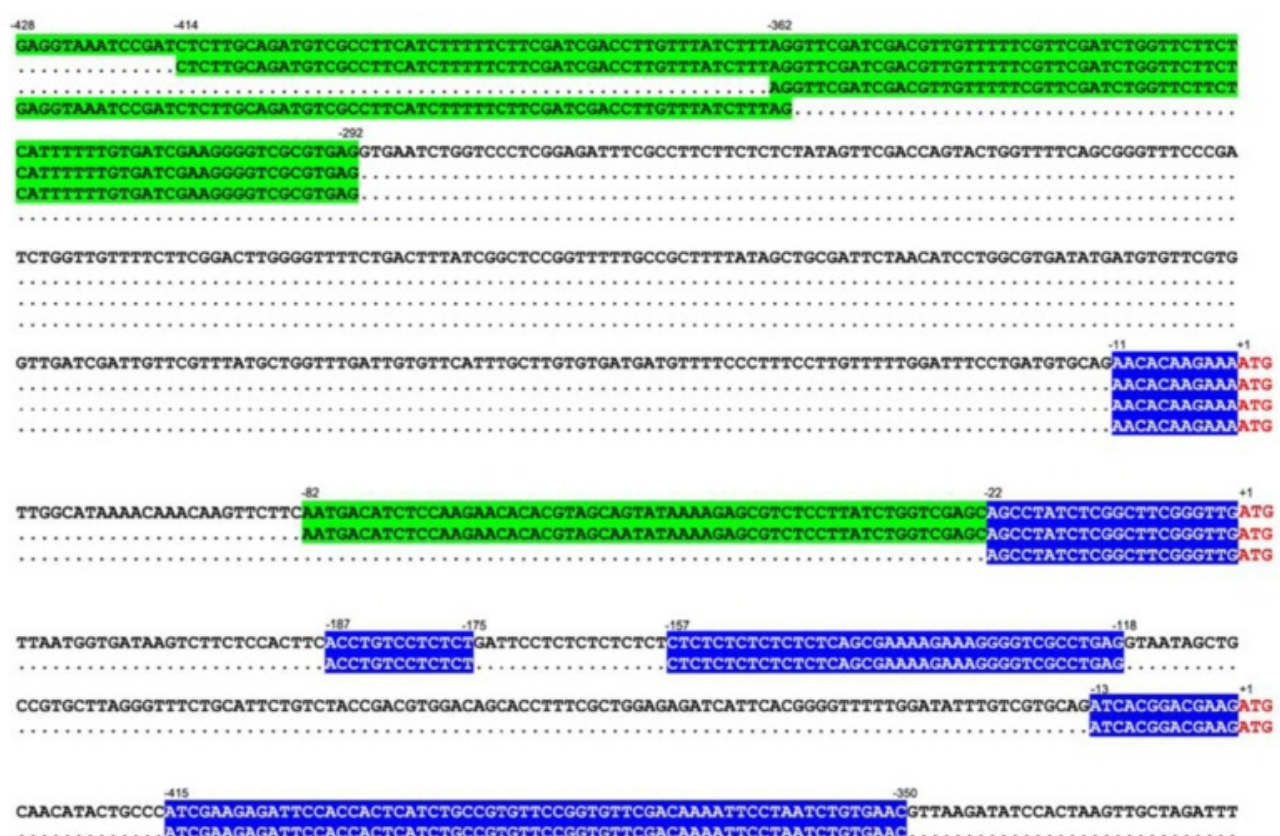

TGCTTTATTGTAAGGATATTTAGTCACACGCCATGCACGAAGACACGTCTTCTTTCTACGCCATGCACGAAGACACGTCATCCTCTTCTTCCGCATCCTCTGCTCTC

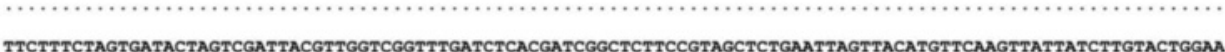

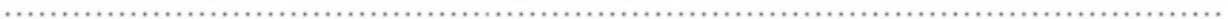
GAGGATTTTGATCTCACTTTCATTGCATANAGTTTTAGAGTTCGTAGTAAGCTGTAAGATCTCATAGTATGATAATTGATATTGGGTGGATCTAATGTGCAGGAACÄTG

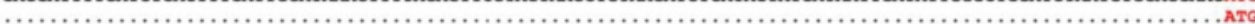

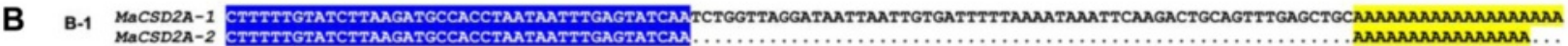

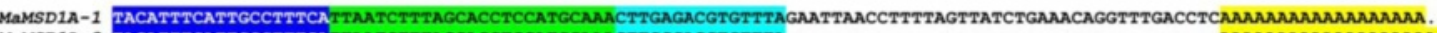

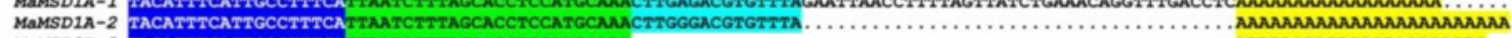

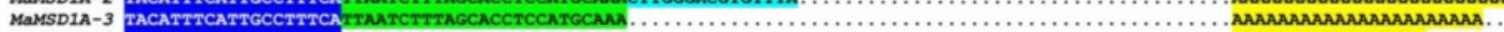

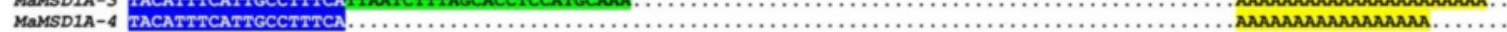
MaMSD1B-1 RGATGCTGTTTCCCTTTCTAATGTATCTACACCATCAGTATGTCACCTGGGTTTTATGTATCATATCTACTTCTTTCAATANAIICACATGAGCAGTAATCCTACCANAAANANANAAAAA

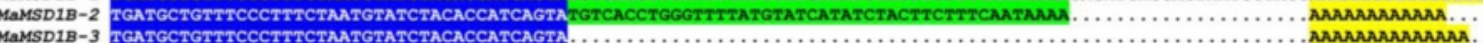
B-4 MaMSD1C-1 FCTAGGTTCCAAGAATCTGTCACTACTTGTTCATGCCCTGTTTFTTTATGCTCTCTACTAGTAGAACTACTGCTTGTGCCAMTGGGGCAACTGGAGTATCACAAAAAAAAAAAAAAAAAAA
MaMSD1C-2

B-5 MaFSD1A-1 GACTATGTAATTGAAGANAGCATACATGTATCCATATGATATTGGCAGCTCTGGTTGAGGTTCTATTTGCCATTTGGTTANTGGAAGTTTTAGTTTGTGTTGGAAAAAAAAAAAAAAAAAA

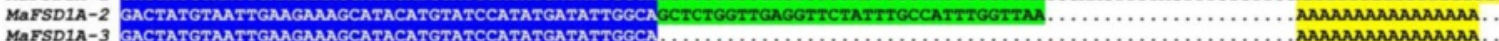

Fig. 1 Alignment of 5'-and 3'-ends of MaSOD cDNAs. a Alignment of 5'-ends of MaSOD cDNAs and their corresponding gDNAs. Coordinates are nucleotide positions relative to the translational start site (ATG). $\mathbf{b}$ Alignment of 3'-ends of MaSOD cDNAs

\section{Gene structures of MaSODs}

The gDNA lengths of MaSODs from the start to stop codons varied between 1807 and 4720 bp (Table 1). A gene-structure map was produced by aligning ORF gDNA sequences with their corresponding cDNA sequences (Fig. 4). Interestingly, the MaSOD gene structures could also be divided into four groups (Ia, Ib, II and III) based on their exon numbers, which is in agreement with the motif-based classification. MaSODs in the same group contained an equal number of exons, with one exception (group III). The four MaCSD1s in group Ia possessed seven exons. However, the size of the first exon in $M a C S D 1 A$ was the same as the one in $A t C S D 3$, while the size of the first exon in the other three $M a C S D 1 s$ (MaCSD1B, $1 C$ and $1 D$ ) was the same as that in AtCSD1. In group Ib, both $M a C S D 2 A$ and $M a C S D 2 B$ had eight exons, just like AtCSD2. Similar to previous reports of AtCSDs and PtCSDs [9], the size of the second exon in MaCSD1s (102 bp) corresponded to that of the second and third exons in MaCSD2s $(62+40 \mathrm{bp})$. This indicates that the inferred exon merge (or split) was shared by monocot and dicot species and should thus to be a defining characteristic of angiosperms. The four MaMSD genes belonging to group II contained six 


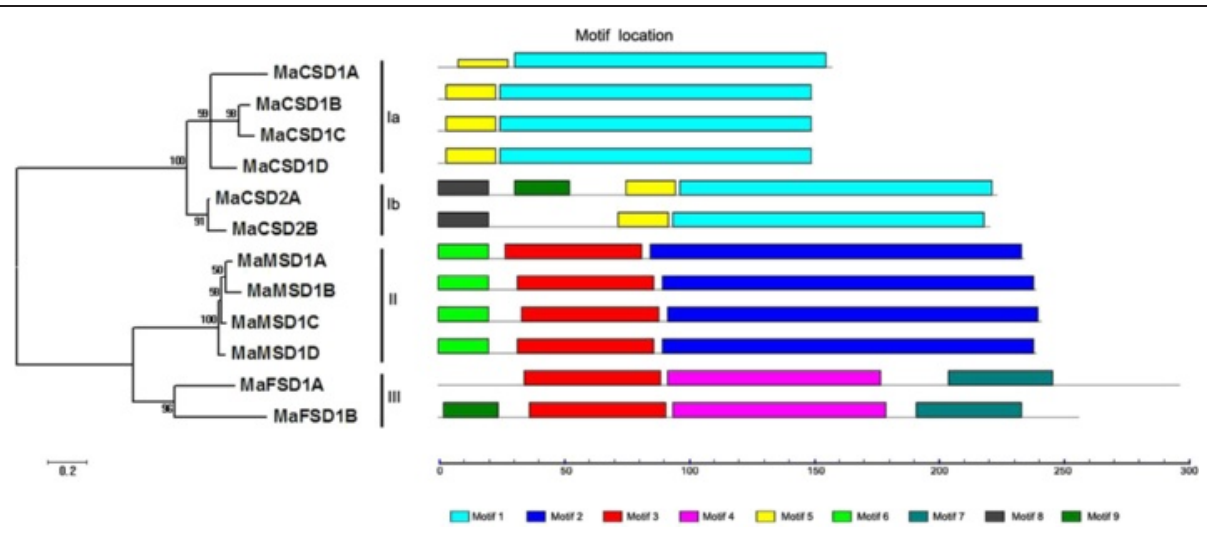

Fig. 2 Conserved motifs and clustering analysis of MaSOD proteins. Clustering tree resulting from the amino acid alignments of MaSODs is shown on the left of the figure. The distribution of conserved motifs among the deduced MaSOD proteins is shown on the right of the figure. Different motif types are marked by different color blocks as indicated at the bottom of the figure. Group la and Ib are copper/zinc superoxide dismutases, Group II are manganese superoxide dismutases, and Group III are iron superoxide dismutases

exons. In contrast to the conserved genomic structures of groups Ia, Ib and II, the genes in group III exhibited different exon-intron organizational patterns. MaFSD1A contained eight exons (one less than its ortholog AtFSD2), while MaFSD1B possessed nine (one more than its ortholog AtFSD3) and MaFSD1B-variant1 harbored seven.

Additionally, many of the introns in banana $S O D$ genes were longer than those in their Arabidopsis homologs, resulting in longer MaSOD gDNAs (Fig. 4). All MaSOD introns were spliced in accordance with the eukaryotic GUAG splice junction site rules [41] with two exceptions: the fourth intron of MaCSD2A and the second of MaMSD1D, which had splice boundaries of GC-AG. Additionally, one intron was found in the 5' UTRs of MaCSD1B and $M a C S D 1 D$ gDNAs, while two were found in the 5' UTR of MaCSD1C gDNA (Fig. 1a-1, a-3 and a-4).

\section{Chromosomal locations and phylogenetic analysis}

The chromosomal locations of MaSOD genes were determined by performing a BLASTn search against the banana genome databases. Eight out of the 11 chromosomes harbored MaSOD genes (Fig. 5), four (chr 2, 3, 8 and 10) of which possessed two MaSOD genes, while the others (chr 4, 7, 9 and 11) contained only one.

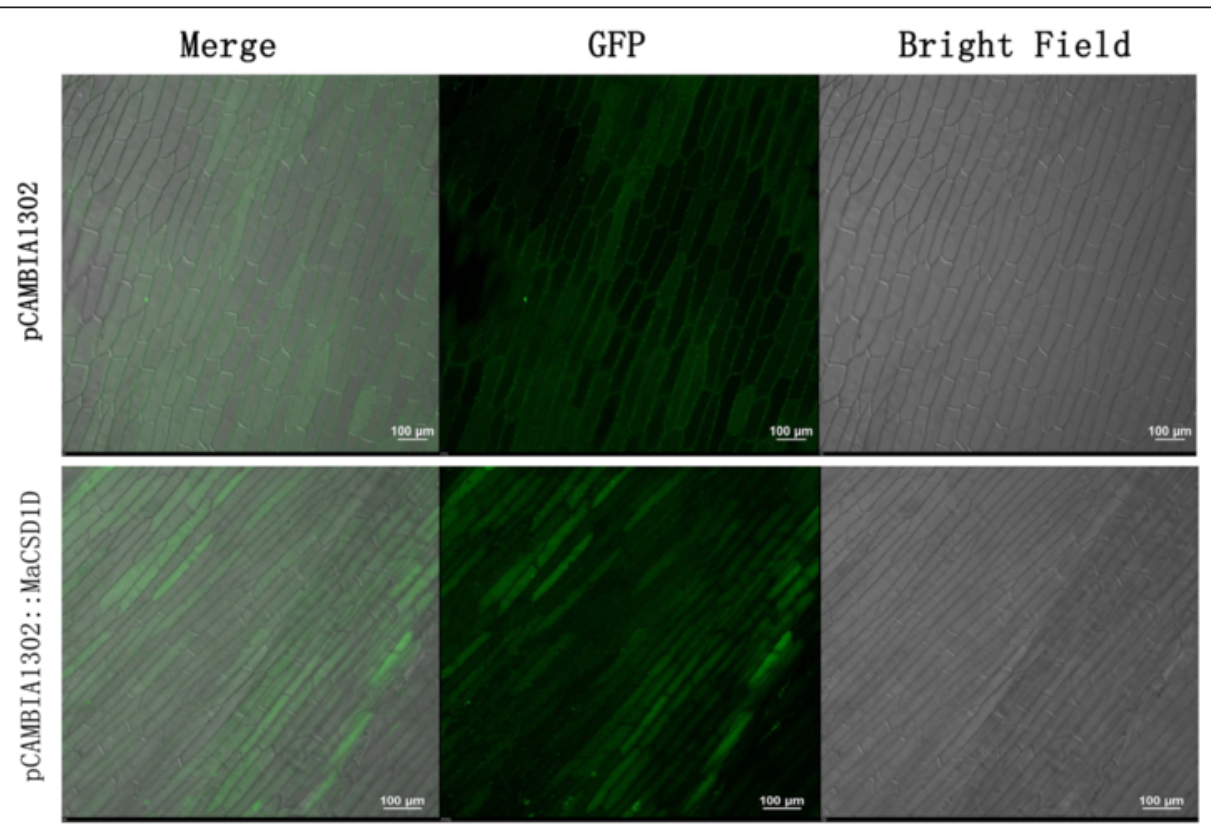

Fig. 3 Subcellular localization analysis of MaCSD1D. The upper panel presents the merged fluorescence, fluorescence and bright filed image of GFP control. The lower panel presents the merged fluorescence, fluorescence and bright filed image of MaCSD1D-GFP 

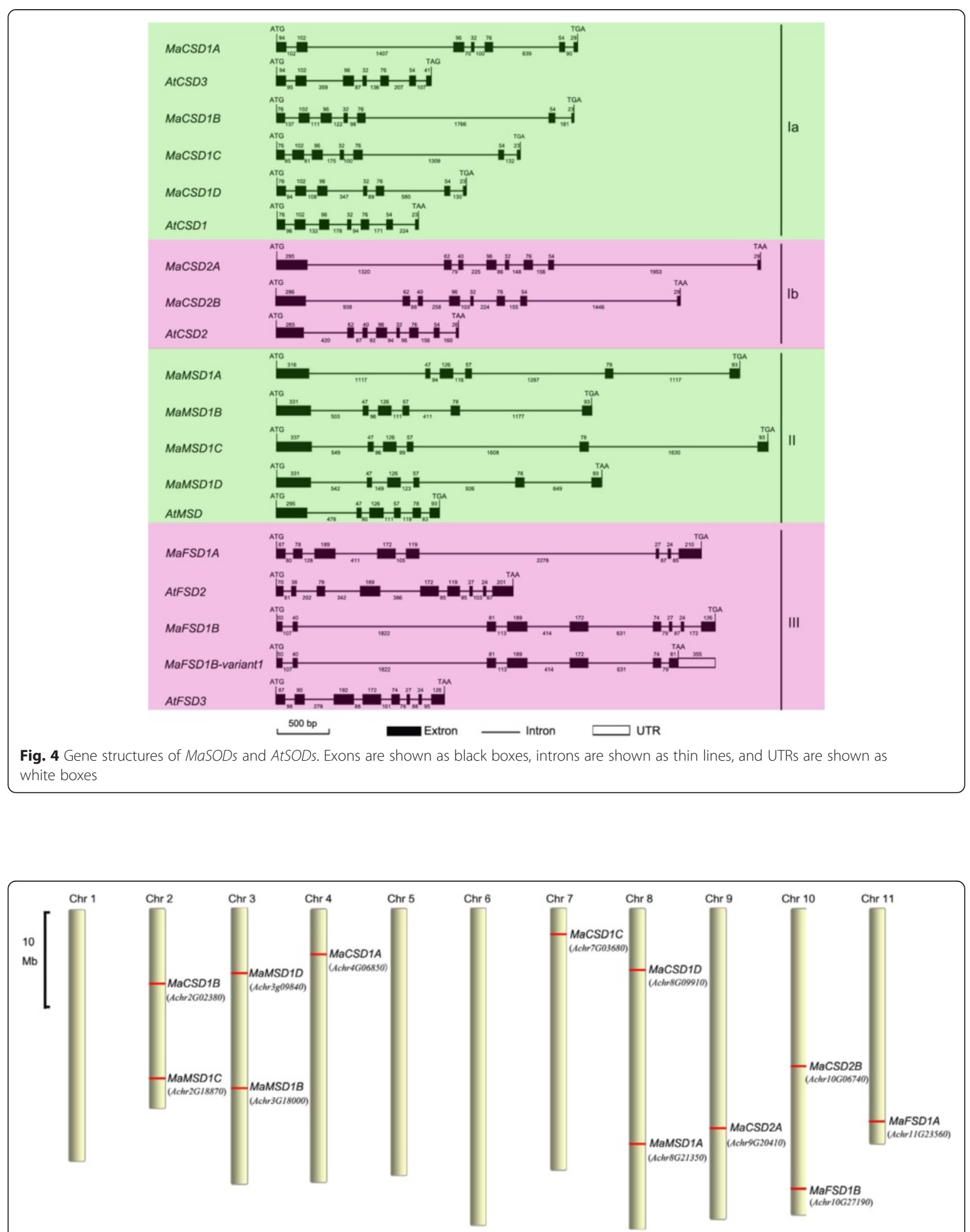

Fig. 5 Chromosomal locations of MaSOD genes. The positions of SOD genes are marked with red lines on the chromosomes. Locus names of "DH-Pahang' SODs are in brackets; for simplicity, the prefix "GSMU_" and suffix "_001" are omitted from each gene name 
To investigate the phylogenetic relationships of MaSODs with other plant SODs, an unrooted phylogenetic tree was constructed from an aligned dataset of MaSODs and 58 homologous SODs from $M$. acuminata var. DH-Pahang, Zea mays, Oryza sativa, Phalaenopsis equestris, A. thaliana, and $P$. trichocarpa. The two major clades of SODs were well supported in this tree (Fig. 6a). All Cu/ZnSODs formed a large clade comprising three subgroups (groups a, b and c). FeSODs were clustered with MnSODs into another large clade, indicating, as previous reports [6], that these two subgroups (groups $\mathrm{d}$ and e) originated from a common ancestor. MaSODs were found in all five subgroups, where they were clustered with 'DH-Pahang' SODs with strong bootstrap support; this close relationship is consistent with their high sequence identities (Additional file 4) and implies that they originated from the same gene.

MaCSD1B, 1C and 1D grouped with AtCSD1 and other cytosolic $\mathrm{Cu} / \mathrm{ZnSODs}$ in group a, while MaCSD1A was placed into another subgroup along with AtCSD3 (group c). The position of these MaCSD1s is consistent with the size of their first exons (Fig. 4), suggesting that two copies of CSD1 existed before the monocot/dicot split, with only one undergoing expansion in banana (to MaCSD1B, $1 C$ and $1 D$ ). MaCSD2A and MaCSD2B clustered in group b with other plant chloroplastic $\mathrm{Cu} / \mathrm{ZnSODs}$.

Interestingly, all four MaMSDs, like PtMSDs and ZmMSDs, were found on a species-specific cluster of the MnSOD clade; this placement indicates that MnSOD gene number amplifications were mainly due to recent WGDs or segmental duplications [9, 31]. Two MaFSDs (MaFSD1A and 1B) fell into different subgroups in group e, only one of which was well supported (85\% bootstrap support). To confirm this result, a small phylogenetic tree limited to FeSODs of group e was constructed (Fig. 6b). In this tree, each MaFSD was separately grouped with FeSOD members from all considered species, revealing the existence of two ancestral FeSOD genes before the monocot/dicot split.

\section{Isolation and bioinformatics analysis of putative MaSOD promoters}

To further understand and determine the regulatory roles of MaSODs under various stresses, the regions upstream of the start codons were isolated by PCR. We obtained a length that varied from 1084 to 2114 bp of 5'-flanking sequences for all $M a S O D$ genes except for $M a C S D 2 B$ (Additional file 2). Potential regulatory cis-elements that were related to stress and light responses are predicted and summarized in Fig. 7. All 11 putative MaSOD promoters possessed typical TATA and CAAT boxes, which are the core elements of the promoters.

As shown in Fig. 7, light-responsive elements accounted for the majority of elements in every MaSOD promoter, excluding MaMSD1A, MaMSD1B and MaMSD1C. There were 25 different types of light-responsive elements present in the 11 banana $S O D$ promoters. Every promoter possessed two to 10 types, which indicated that MaSODs might be differentially regulated when subjected to light. Ten kinds of hormone-responsive regulatory elements were found in the MaSOD promoters, such as ABRE, ERE, CGTAC-motif, TGACG-motif, GARE-motif, P-box, TATC-box, AuxRR-core, TGA-box and TCA element, which were associated with ABA, ethylene, methyl jasmonate (MeJA), GA, auxin and SA responses, respectively. Moreover, six types of stress-responsive regulatory elements, TCA elements, AREs, LTRs, HSEs, TC-rich repeats, MBSs and Box-W1s, with responses to anaerobic induction, low-temperature, heat stress, defense and

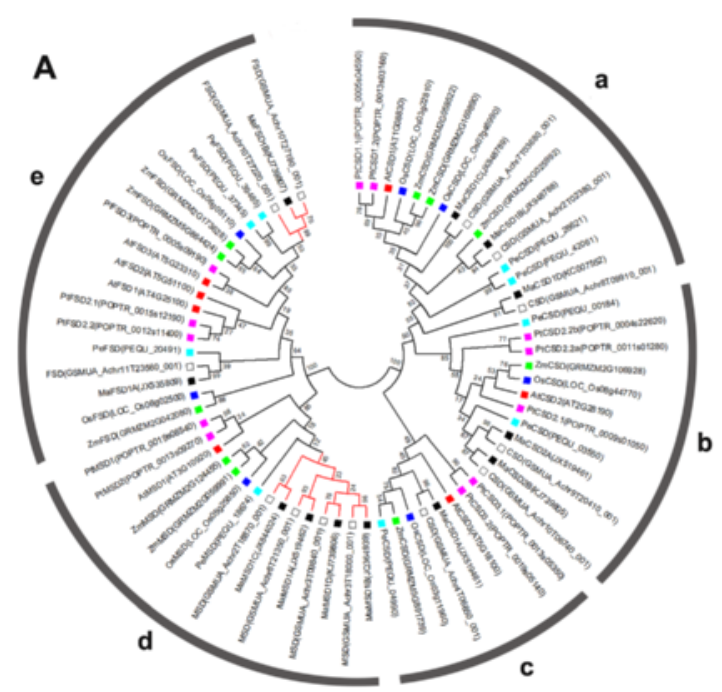

B

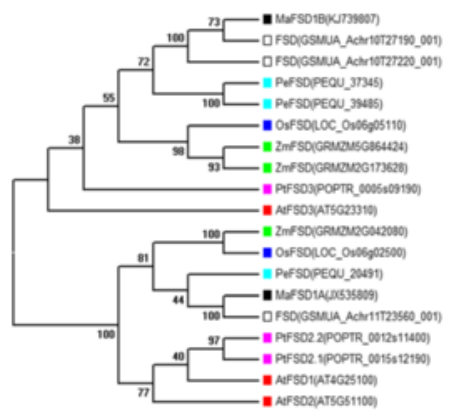

Fig. 6 Phylogenetic trees of the SOD genes from banana and other plants. a A maximum likelihood-based phylogeneitc tree of SOD families. GenBank accession numbers or locus IDs are in brackets. a-e refer to different gene clusters. $\mathbf{b}$ A phylogeneitc tree limited to the FeSODs in group e 


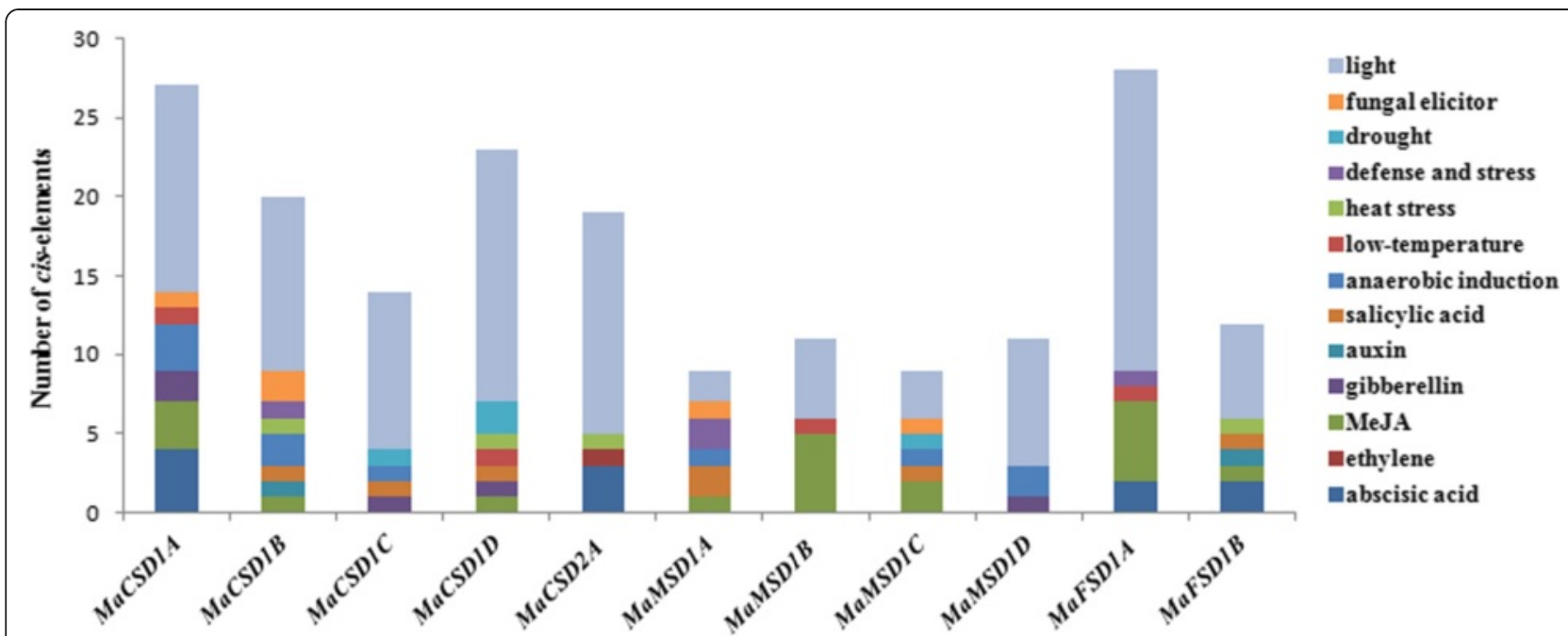

Fig. 7 Cis-element analysis of putative MaSOD promoters related to stress responses. Different cis-elements with the same or similar functions are shown in the same color

stresses, drought inducibility and fungal elicitors, respectively, were identified in the MaSOD promoters. Different types and numbers of regulatory elements were present in distinct MaSOD promoters, indicating that $M a S O D$ genes should have different regulatory mechanisms in response to various stress and hormone treatments.

\section{Expression pattern of MaSOD genes in different tissues} qRT-PCR analysis was performed to assess the expression pattern of MaSOD genes in different organs of 'Tianbaojiao' (Fig. 8). Eleven of the $12 \mathrm{MaSOD}$ genes were expressed in all tested tissues (leaf, pseudostem and root), whereas $M a C S D 2 B$ was expressed only in leaf and root tissues. Different $S O D$-type genes in banana had similar expression patterns. MaCSD1D and MaFSD1A exhibited the highest expression levels in leaves, followed by pseudostems and roots. MaCSD2A and MaFSD1B were expressed strongly in pseudostems, moderately in leaves and weakly in roots. At mRNA level, $M a C S D 1 B$ and MaMSD1B showed their maximum expression in roots, followed by pseudostems and leaves. Expression levels of MaCSD1C and MaMSD1A were low in leaves and pseudostems and, high in roots.

\section{Differential expression of MaSOD genes in response to various abiotic stresses}

The expression patterns of the $12 \mathrm{MaSOD}$ genes as seen by GPCR were detected under cold, heat, drought and $\mathrm{NaCl}$ treatments. The results showed that their expression modes were complex (Fig. 9). During cold treatment, the expression levels of most MaSOD genes exhibited slight variations. However, there were still four $M a C S D$ genes showing obvious differential expression in response to cold stress (MaCSD2A and MaCSD1C were up-regulated, while $M a C S D 1 D$ and $M a C S D 2 B$ were dramatically down-regulated; Fig. 9a). The heat-treatmentresponsive genes were found in all three $S O D$-types. Most of the MaSOD genes (MaCSD1B, MaCSD1D,

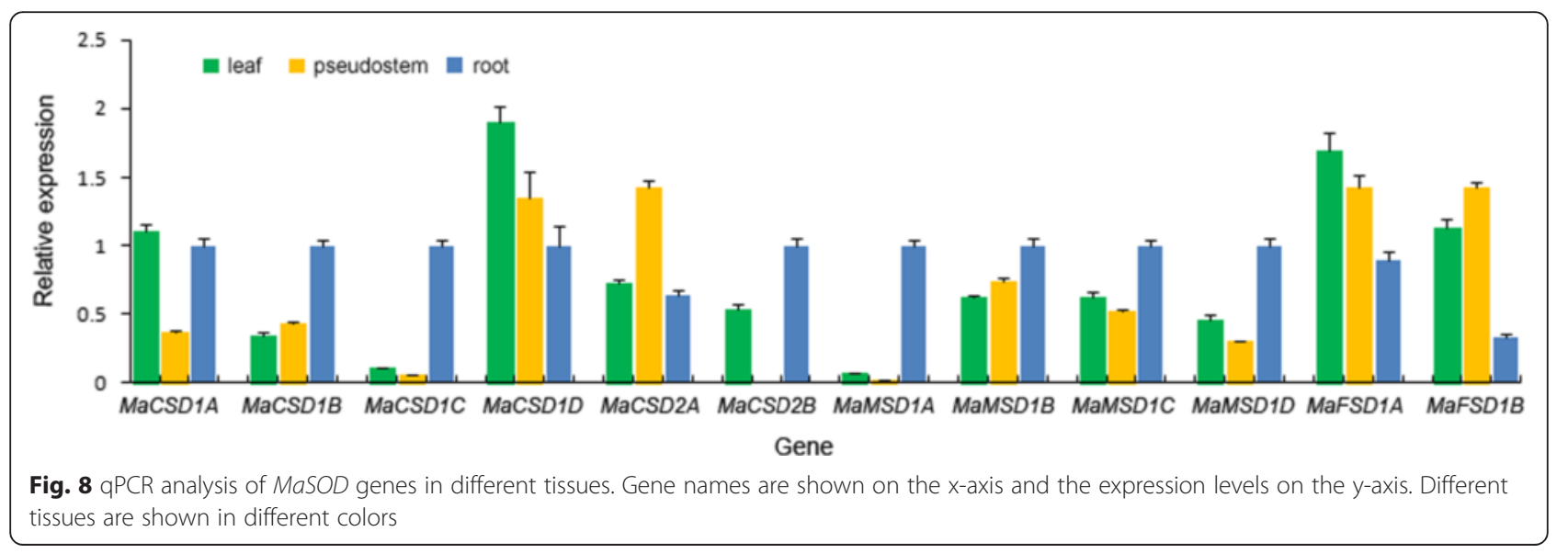



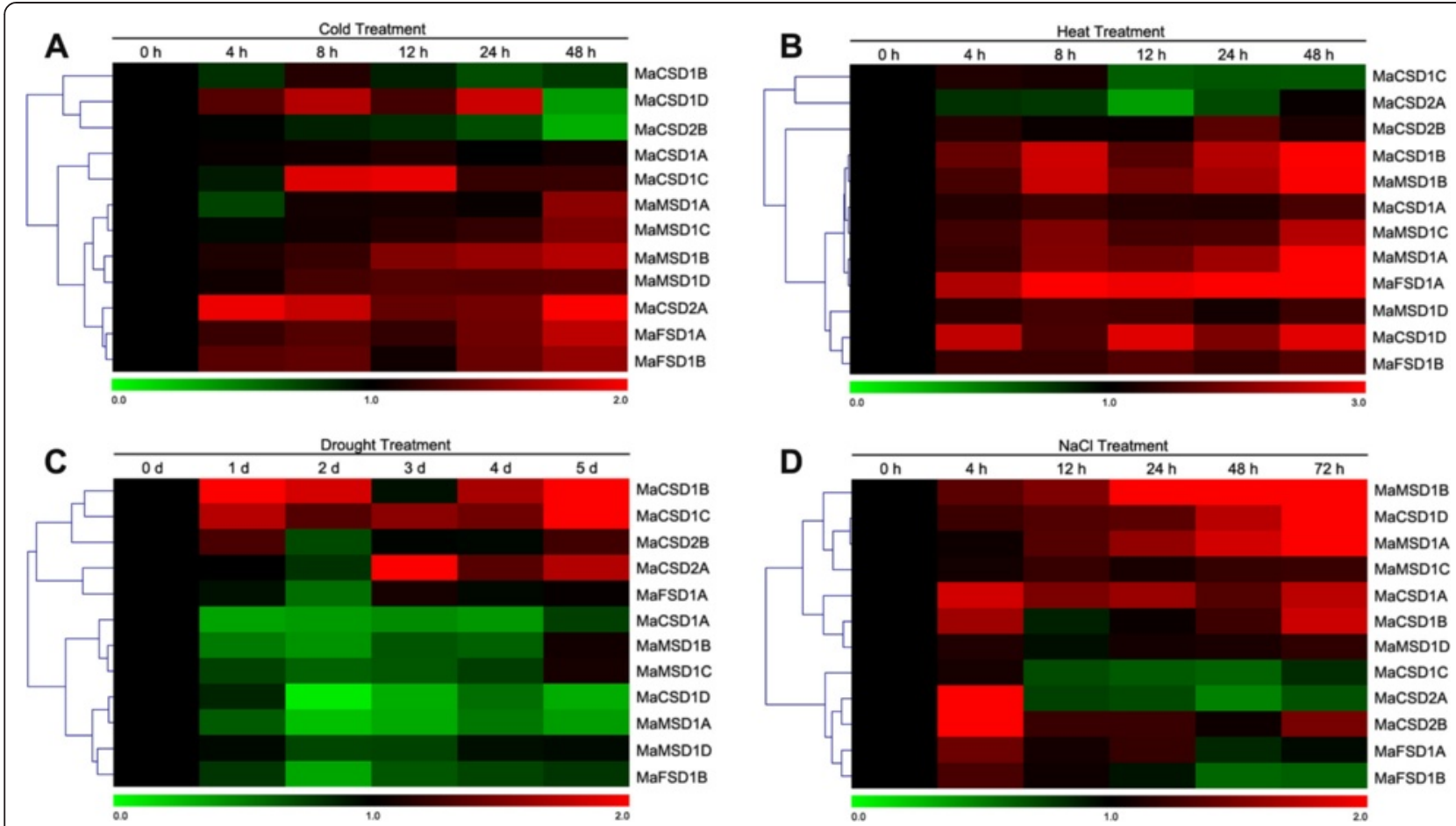

Fig. $9 \mathrm{gPCR}$ analysis of differential gene expression under abiotic treatments. The colors of the bar vary from green to red representing the scale of relative expression levels. Each column represents a sampling time point, and each row represents a MaSOD member. The expression level of the control (at $0 \mathrm{~h} / \mathrm{d}$; marked in black) in every treatment for each gene was used as the rescaled value when calculating the relative expression levels. The clustering results of gene expression patterns are shown on left. a-d refer to different abiotic treatments

MaMSD1A, MaMSD1B, MaMSD1C and MaFSD1A) were increased at the transcriptional levels, except $M a C S D 2 A$, which was down-regulated at $12 \mathrm{~h}$ (Fig. 9b). Obviously, most MaSOD genes were down-regulated in response to drought stress (Fig. 9c). Among them, five members (MaCSD1A, MaCSD1D, MaMSD1A, MaMSD1B and $M a F S D 1 B$ ) exhibited more than 2- to 10 - fold decreases. In contrast, three $\mathrm{Cu} / \mathrm{ZnSOD}$ genes, MaCSD1B, $M a C S D 1 C$ and $M a C S D 2 A$, were strongly induced at $1 \mathrm{~d}, 5$ $\mathrm{d}$ and $3 \mathrm{~d}$, respectively. Under the $\mathrm{NaCl}$ treatment (Fig. 9d), MaCSD1D, MaMSD1A and MaMSD1B shared similar expression patterns, which increased gradually to high levels as the treatment continued. The expression of $M a C S D 1 A$ and $M a C S D 1 B$ was dynamic, increasing quickly at the 4-h point, then decreasing gradually, but finally increasing again. In addition, $M a C S D 1 C, M a C S D 2 A, M a C S D 2 B$ and $M a F S D 1 B$ were first up-regulated at $4 \mathrm{~h}$, and then downregulated over the duration of the treatment.

\section{Differential expression of MaSOD genes in response to hormonal treatments}

The expression of MaSOD genes in response to hormonal stimuli (ABA, GA $\mathrm{GA}_{3}$ IAA and $\mathrm{SA}$ ) was investigated to further analyze the possible functions of MaSODs involved in phytohormone signaling pathways. Most $M a S O D$ genes showed an up-regulation in response to hormonal treatments (Fig. 10). Ten out of the 12 $M a S O D$ genes were up-regulated to different degrees, whereas the other two (MaCSD1C and $M a C S D 2 A)$ were down-regulated under ABA treatment (Fig. 10a). Among the up-regulated genes, $M a C S D 1 D$ exhibited a continuously high-level of transcript abundance over the $48 \mathrm{~h}$ time-course with an 8.9-fold peak at $12 \mathrm{~h}$. During the $\mathrm{GA}_{3}$ treatment, only two genes (MaCSD1D and MaMSD1A) were strongly induced at $4 \mathrm{~h}$ and $48 \mathrm{~h}$, respectively, while the other genes were slightly induced or repressed with no significant changes (Fig. 10b). The IAA treatment dramatically induced three $M a C S D s$ and one MaMSD (MaCSD1A, MaCSD1D, MaCSD2B and MaMSD1A), but didn't obviously repress any member (Fig. 10c). Likewise, the expression levels of most $M a S O D$ genes were up-regulated in response to the SA treatment, but only $M a C S D 1 D$ was greatly transcribed after $24 \mathrm{~h}$ when treated. In addition, the transcription of $M a C S D 1 C$ remained at a low level without a significant change during the treatment time compared with the control (Fig. 10d).

\section{Discussion}

\section{Expanded $S O D$ family in banana}

In this study, we identified 12 SOD genes representing three major types of plant SODs (Cu/ZnSOD, MnSOD 

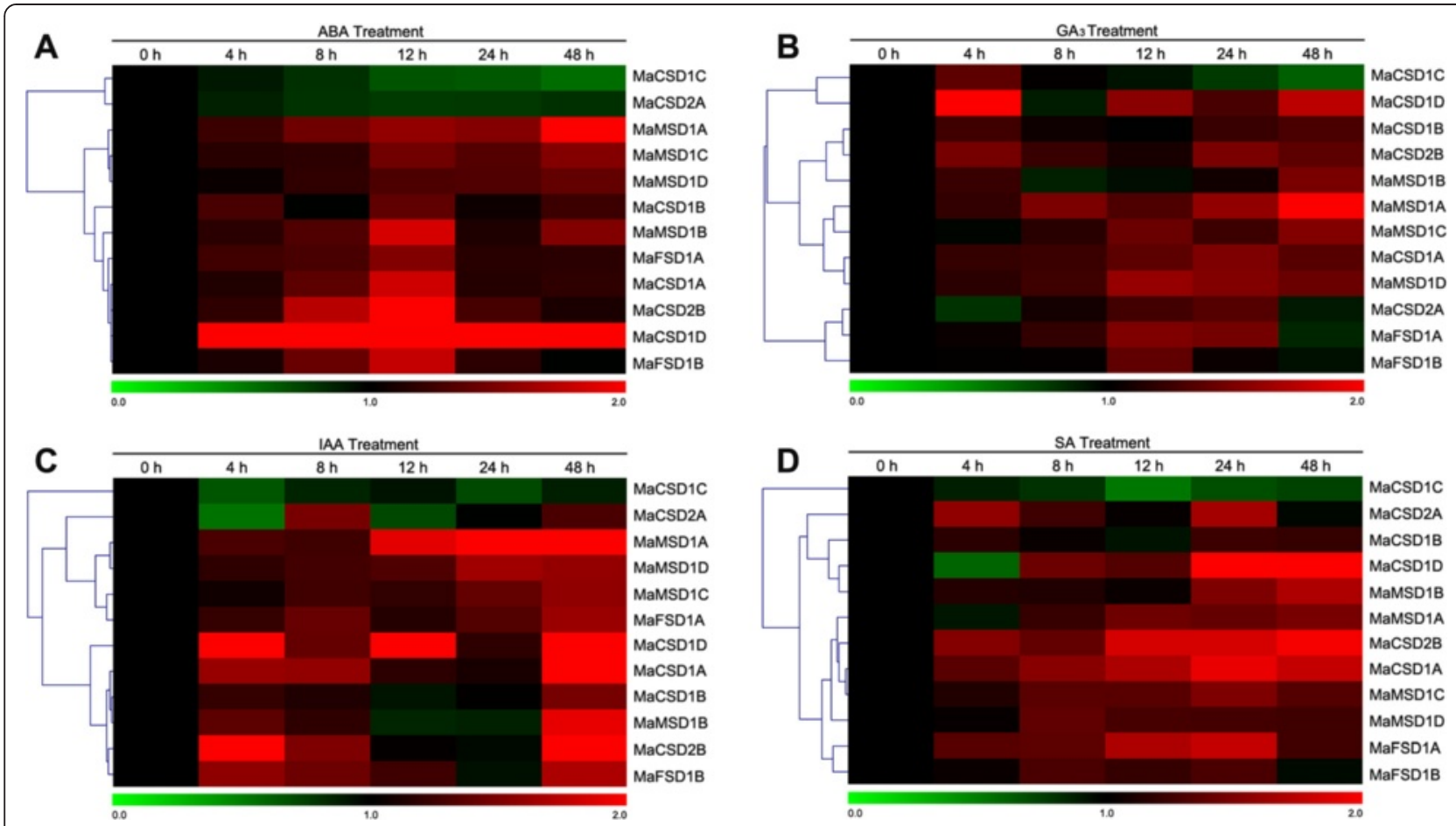

Fig. $10 \mathrm{gPCR}$ analysis of differential gene expression under hormonal treatments. The colors of the bar vary from green to red representing the scale of relative expression levels. Each column represents a sampling time point, and each row represents a MaSOD member. The expression level of the control (at $0 \mathrm{~h} / \mathrm{d}$; marked in black) in every treatment for each gene was used as the rescaled value when calculating the relative expression levels. The clustering results of gene expression patterns are shown on left. a-d refer to different hormonal treatments

and $F e S O D$ ) with 25 distinct transcripts from cultivated banana 'Tianbaojiao' (AAA group). In contrast to wild bananas, which possess two tandem FeSOD genes on chromosome 10, only one $F e S O D$, as assessed by mRNA levels, was isolated from 'Tianbaojiao'. Further sequence analysis revealed that the two tandem FeSOD genes had different putative promoters (1.5 kb upstream of ATG) even thought their ORF and 3' UTR sequences were similar. Forward primers specific to the two promoters and the same reverse primer (FSD1B-ORFR; Additional file 1) were used to amplify the two tandem FeSODs in 'Tianbaojiao'. PCR amplification of each FeSOD using 10 forward primers at different starting positions still yielded only one sequence; this sequence, corresponding to GSMA_ Achr10T27190_001 or ITC1587_Bchr10_T31275, was that of the MaFSD1B gene. Because the two tandem $F e S O D$ genes are present in both the $\mathrm{AA}$ and $\mathrm{BB}$ genome of Musa, the tandem duplication likely predates the A and $\mathrm{B}$ genome independent evolution. We propose that the other FeSOD on chromosome 10 (corresponding to GSMA_Achr10T27220_001 or ITC1587_Bchr10_T31280) was lost in 'Tianbaojiao' over the course of evolution. MaSOD genes had more similar ORF lengths and higher sequence identities with their homologs in the AA genome than in the BB genome (Additional file 4). This observation provides additional evidence to support crosses within diploid $M$. acuminata as the source of the edible triploid M. acuminate [42].

The number of SOD genes varies among monocots (Additional file 8). For instance, the number of distinct $S O D$ genes that have been in silico-identified ranges from 6 in Brachypodium distachyon (3 Cu/ZnSODs, 1 $\mathrm{MnSOD}$ and $2 \mathrm{FeSODs}$ ) and Hordeum vulgare (3 Cu/ $\mathrm{ZnSODs}, 1 \mathrm{MnSOD}$ and $2 \mathrm{FeSODs}$ ) to 7 in O. sativa (4. $\mathrm{Cu} / \mathrm{ZnSODs}, 1 \mathrm{MnSOD}$ and $2 \mathrm{FeSODs}$ ), Sorghum bicolor (4 Cu/ZnSODs, $1 \mathrm{MnSOD}$ and 2 FeSODs) and Setaria italica (4 Cu/ZnSODs, $1 \mathrm{MnSOD}$ and $2 \mathrm{FeSODs}$ ), 9 in $P$. equestris (5 Cu/ZnSODs, $1 \mathrm{MnSOD}$ and 3 FeSODs), 10 in $Z$. mays (5 Cu/ZnSODs, 2 MnSODs and 3 FeSODs) and 18 in Triticum aestivum (10 Cu/ZnSODs, $2 \mathrm{MnSODs}$ and $6 \mathrm{FeSODs}$ ). Excluding the $S O D$ family of hexaploid bread wheat, banana harbors the largest monocot $S O D$ gene family, especially with respect to $M n S O D s$ (four in total). The three whole-genome duplication rounds that have occurred during banana evolution are a major factor responsible for the amplification in gene numbers [25]. However, only five MaSOD members (MaCSD2A and $2 B$, and MaMSD1A and $1 C$ or $1 D$ ) are retained in pairs after banana WGDs, while another five genes (MaCSD1A, MaCSD1D, MaMSD1B, MaFSD1A and MaFSD1B) are 
singletons with the loss of other duplicated copies after WGDs. MaCSD1B, $1 C$ and $1 D$ but not MaCSD1A clustered together in Group a (Fig. 6a) and had identical-sized first exons (Fig. 4) and high sequence similarities (Additional file 6); this indicates that MaCSD1B and $1 C$ were probably derived from recent segmental duplications and shared the same origin with MaCSD1D.

More than one kind of transcript was obtained from seven of the $S O D$ genes in the 'Tianbaojiao' banana. Genes, such as MaCSD2A, MaMSD1A, MaMSD1B, MaMSD1C and MaFSD1A, transcribe two to four different mRNAs as a result of APA. This is not surprising because APA transcripts have already been detected in $\mathrm{Cu}$ / ZnSOD, MnSOD and FeSOD genes from Dimocarpus longan, L. gmelinii, T. aestivum and $Z$. mays [10, 12, 43]. Like APA, AS is a regulatory mechanism to generate multiple transcripts from one single gene. In this study, two kinds of AS occurred in banana SODs. In one, the introns were retained in the 5' UTR region of MaCSD1B mRNA when transcribed, resulting in a normal ORF but a longer 5' UTR. In the other one, the introns resided in the coding region of MaFSD1B, resulting in a premature stop codon, producing a truncated protein (228 aa). Similar observations were made in other plant $\mathrm{Cu}$ / $\mathrm{ZnSOD}$ and $F e S O D$ genes [10, 18, 44]. A previous report in rice suggested that introns that resided in the 5' UTR region were linked to regulating spatially and temporally gene expression [45]. In addition, the rice $F e S O D$ variant with a short ORF is sensitive to low temperatures and its truncated protein is active [46], indicating that a similar variant of the MaFSD1B gene may play a crucial role in the responses to some abiotic stresses. In spite of these, ATSS also provides a regulatory method to generate multiple transcripts. Although a previous study showed that each type of longan $S O D$ genes has multiple transcriptional start site [10], we found only two MaSOD genes (MaCSD1B and MaCSD2A) have ATSSs.

In addition to the above findings, allelic $S O D$ genes (MaCSD1A-1 and MaCSD1A-2, MaMSD1C-1 and $M a M S D 1 C-2)$ with single nucleotide substitutions were detected in the triploid banana 'Tianbaojiao'. This discovery demonstrates that allelic variation exists inside its three A genomes, which is apparently another mechanism promoting RNA and protein diversity in heterozygotes. Furthermore, the sequence polymorphism found between the SOD genes of 'DH-Pahang' and 'Tianbaojiao' (Additional file 4) is direct evidence of sequence variability in the A genomes.

Taken together, our data provide more detailed sequence information and transcriptional regulatory types for banana SODs. Whole genome duplication, segmental duplication and the complex regulation of transcription have contributed to the gene expansion and mRNA diversity of banana SODs.
Specific MaSOD members are involved in different abiotic stress responses

Three types of plant SODs ( $\mathrm{Cu} / \mathrm{ZnSOD}, \mathrm{MnSOD}$ and FeSOD) are all reported to be in involved in ROS scavenging caused by abiotic stress $[8,16]$. The banana $\mathrm{Cu} /$ ZnSOD subfamily comprises six members (MaCSD1A$1 D$ and $M a C S D 2 A-2 B)$. An expression analysis revealed that every $M a C S D$ gene was responsive to at least one abiotic stress treatment performed in this study (cold, heat, drought or salinity) (Fig. 9). Notably, MaCSD1D is the only member that showed obvious expression changes under all four abiotic stresses. Compared with other MaCSD promoters, the MaCSD1D promoter harbored more kinds and numbers of cis-elements involved in abiotic stresses, including one LTR motif (cis-element involved in low-temperature responsiveness), one HSE motif (cis-element involved in heat stress responsiveness) and two MBS motifs (MYB binding site involved in drought-inducibility), which could explain why MaCSD1D showed obvious responses to the four abiotic treatments. This indicates that MaCSD1D might play a predominant antioxidant role in banana. Similarly, various responses to the abiotic stress were also identified in other plant $\mathrm{Cu} / \mathrm{ZnSOD}$ subfamiles [47].

Banana harbors four MnSOD genes (MaMSD1A-1D) with high sequences similarities (80.9-86.5\%), which were in accord with their phylogenitic clustering results. Interestingly, individual MaMSD genes have some distinguishing features from each other, especially in the 5' and 3' UTR regions, indicating that they have evolved over the time and probably underwent modifications to form regulatory diversification under constantly changing environments. This was confirmed by their having different cis-elements involved in stress responses. For instance, two TC-rich repeat motifs (cis-elements involved in defense and stress responsiveness) were present in the MaMSD1A promoter but absent from MaMSD1B, $1 C$ and $1 D$. A qPCR analysis showed that MaMSD1A was responsive to heat, drought and salt stress, while MaMSD1B was responsive to heat and drought stress. MaMSD1C was only responsive to heat stress, and MaMSD1D was not responsive to any stress. This suggested that the four $M n S O D$ genes in banana play distinct roles in scavenging ROS caused by different stimuli.

Additionally, MaSOD members located in the same subcellular department also exhibited different expression patterns. The expression of chloroplastic $M a C S D 2 B$ decreased during the cold treatment while that of $M a C S D 2 A$ increased, and vice versa during the $\mathrm{NaCl}$ treatment. Likewise, drought up-regulated the expression of cytosolic MaCSD1B and MaCSD1C, whereas it down-regulated MaCSD1A and MaCSD1D. MaMSD1A and $M a M S D 1 B$ exhibited increased expression levels under $\mathrm{NaCl}$ stress while the other two members 
(MaMSD1C and MaMSD1D) showed little change in expression. Our data also show that heat stress represses the expression of chloroplastic MaCSD2A but strongly induces chloroplastic MaFSD1A. In transgenic tobacco, the over-expression of chloroplastic FeSOD suppressed the expression of both chloroplastic and cytosolic $\mathrm{Cu} /$ ZnSODs, and the authors proposed that chloroplastic FeSOD over-expression interfered with a signal pathway regulating the $\mathrm{Cu} / \mathrm{ZnSODs}$ through a low superoxide radical concentration [48]. Therefore, we hypothesize that there may exist some inter and cross-family signal pathways regulating the expression of MaSODs under various environmental stresses.

In addition, the promoters of MaCSDs and MaFSDs contain large amounts of light responsive cis-elements of 5 to 10 different types (Fig. 7). Kurepa et al. detected an increased accumulation of both $\mathrm{Cu} / \mathrm{ZnSOD}$ and FeSOD transcripts in tobacco when exposed to light stress [49]. Similar observations were also detected in Arabidopsis and rice [7]. Tobacco plants harboring an over-expressed $\mathrm{Cu}$ / $\mathrm{ZnSOD}$ gene from pea exhibited an increased tolerance against high light [50]. Therefore, we propose that $M a C S D s$ and MaFSDs might participate in light responses.

\section{MaSODs probably participate in phytohormone signaling pathways}

Hormone-responsive transcription factors are known to act via combination with their corresponding cis-elements in the promoter to regulate the expression of target genes during various stresses [51]. The PlantCARE database predicted that four MaSOD promoters (MaCSD1A, $M a C S D 2 A, M a F S D 1 A, M a F S D 1 B)$ contained two to four ABREs, a cis-acting element involved in the abscisic acid responsiveness, indicating that these genes probably participate in ABA responses. The mRNA levels of $M a C S D 1 A, M a F S D 1 A$ and MaFSD1B were induced to 1.8-fold, 1.5-fold and 1.8-fold, respectively, at $12 \mathrm{~h}$ under ABA treatment, whereas the transcripts of $M a C S D 2 A$ were slightly reduced over the treatment's time course. Moreover, the expression of three other members (MaCSD1D, MaCSD2B and MaMSD1A), which had no ABREs, showed $>2.0$-fold expression inductions during the ABA treatment, suggesting that there are other regulation mechanisms responding to $\mathrm{ABA}$. In the recent study, ABA was shown to regulate the expression of miR398 [52], which was negatively correlated with that of its target genes $(\mathrm{Cu} / \mathrm{ZnSODs})[19,53]$. MiR398 was also reported to exist in banana [25], and a sequence analysis found binding sites for miR398 in the mRNAs of MaCSD2A, but not in $M a C S D 1 A$ mRNA, which may explain why the $M a C S D 2 A$ was down-regulated under ABA treatment. We therefore proposed that the expression of MaSOD genes in response to ABA may be synergistically mediated by ABREs and miRNAs. In addition to ABA, tobacco $S O D$ genes were also reported to be responsive to auxin, gibberellin A and other substances [54]. In this study, we predicted using the PlantCARE database that there were eight other hormone-responsive cis-elements (ERE, CGTA-motif, TGACG-motif, GARE-motif, P-box, TATCbox, AuxRR-core and TCA-element) located 5'-upstream of MaSODs. We confirmed their transcriptional regulation under $\mathrm{GA}_{3}$, IAA and SA treatments using qPCR. Furthermore, $\mathrm{H}_{2} \mathrm{O}_{2}$ produced in the dismutation reaction by SODs is also a signal molecular that can interact with phytohormones to affect various metabolic processes in the cell under stress [55-57]. We hypothesize that MaSODs probably participate in phytohormone signaling pathways.

\section{Conclusions}

Banana harbors $12 S O D$ genes, including three types of plant SODs (Cu/ZnSOD, MnSOD and FeSOD). Whole genome duplication, segmental duplication, APA, AS and ATSS have contributed to the gene expansion and mRNA diversity of banana SODs. The 12 MaSODs were distributed on eight out of the 11 banana chromosomes. Based on structural characteristics, the 12 MaSODs were divided into four groups. Promoter sequence analyses revealed that there were many abiotic and hormonal-responsive ciselements in the $5^{\prime}$ upstream regions of the MaSODs, but distinct members harbored different types and numbers, which suggested that the $12 \mathrm{MaSODs}$ were differentially regulated. A qPCR analysis revealed that distinct MaSOD genes exhibited different expression patterns in response to abiotic and hormonal stresses, which indicated that specific MaSOD members play roles in different aspects of banana abiotic stress tolerance and hormonal signaling pathway.

\section{Additional files}

Additional file 1: Table S1. Oligonucleotide primers used for cloning MaSOD genes. (PDF $81 \mathrm{~kb}$ )

Additional file 2: Table S2. Oligonucleotide primers used for cloning the 5'- flanking regions of MaSOD genes. (PDF $102 \mathrm{~kb}$ )

Additional file 3: Table $\mathbf{S 3}$. Specific primers used for quantitative real-time PCR. (PDF $62 \mathrm{~kb}$ )

Additional file 4: Table S4. Information of SOD genes from the wild and cultivated bananas. (PDF $13 \mathrm{~kb}$ )

Additional file 5: Figure S1. Sequence alignment of MaFSD1B and MaFSD1B-variant1. (PDF $211 \mathrm{~kb}$ )

Additional file 6: Table S5. Pairwise identity of the coding region CDNAs and deduced amino acids of MaSOD genes. (PDF $13 \mathrm{~kb}$ )

Additional file 7: Table S6. Motif sequences of MaSODs identified by MEME tools. (PDF $14 \mathrm{~kb}$ )

Additional file 8: Table S7. Potential functional SOD family genes identified in silico in monocot plants. (PDF $13 \mathrm{~kb}$ )

\section{Abbreviations}

SOD: Superoxide dismutase; CSD or Cu/ZnSOD: Copper/zinc superoxide dismutase; MSD or MnSOD: Manganese superoxide dismutase; FSD or 
FeSOD: Iron superoxide dismutase; APA: Alternative polyadenylation; AS: Alternative splicing; ATSS: Alternative transcription site; ABA: Abscisic acid; $G_{3}$ : Gibberellin $A_{3}$; IAA: Indole-3-acietic acid; SA: Salicylic acid; ROS: Reactive oxygen species; PKW: Musa balbisiana var. Pisang Klutuk Wulang; CAC: Clathrin adaptor complexes medium; WGD: Whole genome duplication.

\section{Competing interests}

The authors declare that they have no competing interests.

\section{Authors' contributions}

$Z \mathrm{~L}, \mathrm{YL}$ and $\mathrm{XF}$ designed and prepared the manuscript. XF performed the genes identification and sequences analysis. XF, GL and $C L$ carried out the experiment of subcellular localization and gene expression assays. All authors read and approved the final manuscript.

\section{Acknowledgements}

We are grateful to Dr. Min Kyaw Thu (Department of botany, Maubin University) for his grammar check. This work was supported by the earmarked Fund for China Agriculture Research System (CARS-32-11) and the Fujian Provincial Science and Technology Platform Construction Project (2008 N2001).

Received: 17 December 2014 Accepted: 9 October 2015

Published online: 20 October 2015

\section{References}

1. Shyam S, Yadav, Robert J: Crop adaptation to climate change. [http:// onlinelibrary.wiley.com/doi/10.1002/9780470960929.ch29/summary:426-435].

2. Karuppanapandian T, Moon J-C, Kim C, Manoharan K, Kim W. Reactive oxygen species in plants: their generation, signal transduction, and scavenging mechanisms. Aust J Crop Sci. 2011;5(6):709-25.

3. Karuppanapandian T, Wang HW, Prabakaran N, Jeyalakshmi K, Kwone M, Manoharan $\mathrm{K}$, et al. 2,4-dichlorophenoxyacetic acid-induced leaf senescence in mung bean (Vigna radiata L. Wilczek) and senescence inhibition by co-treatment with silver nanoparticles. Plant Physiol Biochem. 2011;49(2):168-77.

4. Lee S-H, Ahsan N, Lee K-W, Kim D-H, Lee D-G, Kwak S-S, et al. Simultaneous overexpression of both CUZn superoxide dismutase and ascorbate peroxidase in transgenic tall fescue plants confers increased tolerance to a wide range of abiotic stresses. J Plant Physiol. 2007;164(12):1626-38.

5. Wang B-Q, Chen X, Xiang C-B. Arabidopsis late-flowering mutants with elevated SOD activities show enhanced tolerance to abiotic stresses. Chin Bull Bot. 2007;24(5):572-80.

6. Alscher RG, Erturk N, Heath LS. Role of superoxide dismutases (SODs) in controlling oxidative stress in plants. J Exp Bot. 2002;53(372):1331-41.

7. Pilon M, Ravet K, Tapken W. The biogenesis and physiological function of chloroplast superoxide dismutases. Biochim Biophys Acta. 1807;2011:989-98.

8. Kliebenstein DJ, Monde R-A, Last RL. Superoxide dismutase in Arabidopsis: an eclectic enzyme family with disparate regulation and protein localization. Plant Physiol. 1998;118(2):637-50.

9. Molina-Rueda JJ, Tsai CJ, Kirby EG: The populus superoxide dismutase gene family and its responses to drought stress in transgenic poplar overexpressing a pine cytosolic glutamine synthetase (GS1a). Plos One 2013, 8(2): doi:10.1371/ journal.pone.0056421.

10. Lin Y-L, Lai Z-X. Superoxide dismutase multigene family in longan somatic embryos: a comparison of CUZn-SOD, Fe-SOD, and Mn-SOD gene structure, splicing, phylogeny, and expression. Mol Breed. 2013;32(3):595-615.

11. Zhu D, Scandalios JG. Maize mitochondrial manganese superoxide dismutases are encoded by a differentially expressed multigene family. Proc Natl Acad Sci U S A. 1993;90(20):9310-4.

12. Baek K-H, Skinner DZ, Ling $P$, Chen $X$. Molecular structure and organization of the wheat genomic manganese superoxide dismutase gene. Genome. 2006:49(3):209-18.

13. Wang $B$, Lüttge $U$, Ratajczak R. Specific regulation of SOD isoforms by $\mathrm{NaC}$ and osmotic stress in leaves of the C3 halophyte Suaeda salsa L. J Plant Physiol. 2004;161(3):285-93.

14. Asensio AC, Gil-Monreal M, Pires L, Gogorcena Y, Aparicio-Tejo PM, Moran JF. Two Fe-superoxide dismutase families respond differently to stress and senescence in legumes. J Plant Physiol. 2012;169(13):1253-60.

15. Gómez J, Hernández J, Jiménez A, Río LD, Sevilla F. Differential response of antioxidative enzymes of chloroplasts and mitochondria to long-term $\mathrm{NaC}$ stress of pea plants. Free Radic Res. 1999;31(Suppl):S11-18.
16. Wu G, Wilen RW, Robertson AJ, Gusta LV. Isolation, chromosomal localization, and differential expression of mitochondrial manganese superoxide dismutase and chloroplastic copper/zinc superoxide dismutase genes in wheat. Plant Physiol. 1999;120(2):513-20.

17. Baeka K-H, Skinner DZ. Alteration of antioxidant enzyme gene expression during cold acclimation of near-isogenic wheat lines. Plant Sci. 2003;165(6):1221-7.

18. Srivastava V, Srivastava MK, Chibani K, Nilsson R, Rouhier N, Melzer M, et al. Alternative splicing studies of the reactive oxygen species gene network in populus reveal two isoforms of high-isoelectric-point superoxide dismutase. Plant Physiol. 2009;149(4):1848-59.

19. Lu Y, Feng Z, Bian L, Xie H, Liang J. miR398 regulation in rice of the responses to abiotic and biotic stresses depends on CSD1 and CSD2 expression. Funct Plant Biol. 2011;38(1):44-53.

20. Myouga F, Hosoda C, Umezawa T, lizumi H, Kuromori T, Motohashi R, et al. A heterocomplex of iron superoxide dismutases defends chloroplast nucleoids against oxidative stress and is essential for chloroplast development in Arabidopsis. Plant Cell. 2008;20(11):3148-62.

21. Prashanth SR, Sadhasivam V, Parida A. Over expression of cytosolic copper/ zinc superoxide dismutase from a mangrove plant Avicennia marina in indica Rice var Pusa Basmati-1 confers abiotic stress tolerance. Transgenic Res. 2008;17(2):281-91.

22. Zhang D-Y, Yang H-L, Li X-S, Li H-Y, Wang Y-C. Overexpression of Tamarix albiflonum TaMnSOD increases drought tolerance in transgenic cotton. Mol Breed. 2014;34(1):1-11.

23. Zhou B-Y, Liang L-F, Huang H-B, Wang Z-H. Effects of low temperature and paclobutrazol on superoxide dismutase and abscisic acid of bananas (Musa spp.). Acta Horticult Sin. 1995;22(4):331-5.

24. Yang Q-S, Wu J-H, Li C-Y, We Y-R, Sheng O, Hu C-H, et al. Quantitative proteomic analysis reveals that antioxidation mechanisms contribute to cold tolerance in plantain (Musa paradisiaca L.; ABB Group) seedlings. Mol Cell Proteomics. 2012;11(12):1853-69.

25. D'Hont A, Denoeud F, Aury J-M, Baurens F-C, Carreel F, Garsmeur O, et al. The banana (Musa acuminata) genome and the evolution of monocotyledonous plants. Nature 2012, 488(7410): doi:10.1038/nature11241.

26. Davey MW, Gudimella R, Harikrishna JA, Sin LW, Khalid N, Keulemans J. A draft Musa balbisiana genome sequence for molecular genetics in polyploid, inter- and intra-specific Musa hybrids. BMC Genomics. 2013;14:683.

27. Zhang M-X, Lai Z-X, He S-L, Huang Y-J, Lin Y-L, Wu J-S. Development of a transgenic acceptor system for banana. J Fujian Agriculture For Univ. 2006;35(5):509-14.

28. Stewart Jr CN, Via LE. A rapid CTAB DNA isolation technique useful for RAPD fingerprinting and other PCR applications. Biotechniques. 1993;14(5):748-50.

29. Droc G, Lariviere D, Guignon V, Yahiaoui N, This D, Garsmeur O, et al. The Banana Genome Hub. Database 2013:bat035.doi:10.1093/database/bat1035.

30. Gasteiger E, Hoogland C, Gattiker A, Duvaud S, Wilkins MR, Appel RD, et al. Protein identification and analysis tools on the ExPASy server. In: John M, Walker, editors. The Proteomics Protocols Handbook. New Jersey: Humana Press; 2005. p. 571-607.

31. Lee $T-H$, Tang $H$, Wang $X$, Paterson AH. PGDD: a database of gene and genome duplication in plants. Nucleic Acids Res. 2013;41(D1):D1152-8.

32. Lescot M, Déhais $P$, Thijs $G$, Marchal $K$, Moreau $Y$, Peer $Y V D$, et al. PlantCARE, a database of plant cis-acting regulatory elements and a portal to tools for in silico analysis of promoter sequences. Nucleic Acids Res. 2002:30(1):325-7.

33. Emanuelsson $\mathrm{O}$, Nielsen $\mathrm{H}$, Heijne GV. ChloroP, a neural network-based method for predicting chloroplast transit peptides and their cleavage sites. Protein Sci. 1999;8(5):978-84.

34. Claros MG, Vincens P. Computational method to predict mitochondrially imported proteins and their targeting sequences. Eur J Biochem. 1996:241(3):779-86.

35. Liu H-Y, Feng D-R, Liu B, He Y-M, Wang H-B, Wang J-F. Studies on subcellular localization of MpASR in onion epidermal cells mediated by agrobacterium. J Trop Subtrop Bot. 2009;17(3):218-22.

36. Tamura K, Peterson D, Peterson N, Stecher G, Nei M, Kumar S. MEGA5: molecular evolutionary genetics analysis using maximum likelihood, evolutionary distance, and maximum parsimony methods. Mol Biol Evol. 2011;28(10):2731-9.

37. Chen L, Zhong H-Y, Kuang J-F, Li J-G, Lu W-J, Chen J-Y. Validation of reference genes for RT-qPCR studies of gene expression in banana fruit under different experimental conditions. Planta. 2011;234(2):377-90. 
38. Feng X, Lai Z-X. Cloning and bioinformatics analysis of Cu/Zn-SOD genes from both 'Tianbao' banana in Fujian and the wild banana in Fuzhou. In: Zongyong Jian, Ganjun Yi, Ahmad Yacob, editors. 5th International Symposium on Tropical and Subtropical Fruits. Guangzhou: ISHS; 2012. p. 60.

39. Katyshev Al, Konstantinov YM, Kobzev VF. Characterization of Mn- and Cu/ Zn-containing superoxide dismutase gene transcripts in Larix gmelinii. Mol Biol. 2006;40(2):372-4.

40. Parker MW, Blake CCF. Iron- and manganese-containing superoxide dismutases can be distinguished by analysis of their primary structures. FEBS Lett. 1988;229(2):377-82.

41. Breathnach $R$, Chambon P. Organization and expression of eucaryotic split genes coding for proteins. Annu Rev Biochem. 1981;50:349-83.

42. Simmonds NW, Shepherd K. The taxonomy and origins of the cultivated bananas. J Linn Soc Lon Bot. 1955;55(359):302-12.

43. Katyshev Al, Rogozin IB, Konstantinov YM. Identification of new superoxide dismutase transcripts in plants by est analysis: Alternative polyadenylation and splicing events. In: Kolchanov N, Hofestadt R, editors. Proceedings of the Fifth International Conference on Bioinformatics of Genome Regulation and Structure. Novosibirs: Russian Acad Sci Siberian Branch; 2006. p. 1:61-64.

44. Lian C-L, Lai Z-X, Lu B-G, Lin Y-L, Feng X. Cloning and bioinformatics analysis of $\mathrm{Fe}-\mathrm{SOD}$ gene from embryogenic callus of an ancient litchi tree. Chin J Trop Crops. 2014;35(1):74-81.

45. Kitagawa N, Washio T, Kosugi S, Yamashita T, Higashi K, Yanagawa H, et al. Computational analysis suggests that alternative first exons are involved in tissue-specific transcription in rice (Oryza sativa). Bioinformatics. 2005:21(9):1758-63.

46. Wang F, Wang H, Liu B, Wang J. Cloning and characterization of a novel splicing isoform of the iron-superoxide dismutase gene in rice (Oryza sativa L.). Plant Cell Rep. 2006;24(12):734-42.

47. Tsang EWT, Bowler C, Herouart D, Camp W, Villarroel R, Genetello C, et al. Differential regulation of superoxide dismutases in plants exposed to environmental stress. Plant Cell. 1991;3(8):783-92.

48. Camp WV, Capiau K, Montagu MV, Inzé D, Slooten L. Enhancement of oxidative stress tolerance in transgenic tobacco plants overproducing Fe-superoxide dismutase in chloroplasts. Plant Physiol. 1996;112(4):1703-14.

49. Kurepa J, Montagu MV, Inzé D. Expression of $\operatorname{sod} C p$ and $\operatorname{sodB}$ genes in Nicotiana tabacum: effects of light and copper excess. J Exp Bot. 1997;48(12):2007-14

50. Gupta AS, Heinen JL, Holaday AS, Burke JJ, Allen RD. Increased resistance to oxidative stress in transgenic plants that overexpress chloroplastic $\mathrm{Cu} / \mathrm{Zn}$ superoxide dismutase. Proc Natl Acad Sci U S A. 1993;90(4):1629-33.

51. Wang S, Bai Y, Shen C, Wu Y, Zhang S, Jiang D, et al. Auxin-related gene families in abiotic stress response in Sorghum bicolor. Funct Integr Genomics. 2010;10(4):533-46.

52. Jia X, Wang W-X, Ren L, Chen Q-J, Mendu V, Willcut B, et al. Differential and dynamic regulation of miR398 in response to $A B A$ and salt stress in Populus tremula and Arabidopsis thaliana. Plant Mol Biol. 2009;71(1-2):51-9.

53. Sunkar R, Kapoor A, Zhu J-K. Posttranscriptional induction of two Cu/Zn superoxide dismutase genes in Arabidopsis is mediated by downregulation of miR398 and important for oxidative stress tolerance. Plant Cell. 2006;18(8):2051-65.

54. Kurepa J, Herouart D, Montagu MV, Inzé D. Differential expression of CuZnand Fe-superoxide dismutase genes of tobacco during development, oxidative stress, and hormonal treatments. Plant Cell Physiol. 1997:38(4):463-70

55. Chen Z, Silva H, Klessig DF. Active oxygen species in the induction of plant systemic acquired resistance by salicylic acid. Science. 1993;262(5141):1883-6.

56. Pei Z-M, Murata Y, Benning G, Thomine S, Klusener BK, Allen GJ, et al. Calcium channels activated by hydrogen peroxide mediate abscisic acid signalling in guard cells. Nature. 2000;406(6797):731-4.

57. Jiang $M$, Zhang J. Effect of abscisic acid on active oxygen species, antioxidative defence system and oxidative damage in leaves of maize seedlings. Plant Cell Physiol. 2001;42(11):1265-73. 